\title{
Co-research with older people: a systematic literature review
}

\author{
Hayley James ${ }^{1 \star}$ (D) and Tine Buffel ${ }^{2}$ \\ ${ }^{1}$ School of Social Policy, Social Work and Social Justice, University College Dublin, Dublin, Ireland and \\ ${ }^{2}$ The Manchester Institute for Collaborative Research on Ageing (MICRA), The University of Manchester, \\ Manchester, UK \\ *Corresponding author. Email: hayley.james@ucd.ie
}

(Accepted 22 December 2021)

\begin{abstract}
Interest in co-research with older adults has grown in the past 20 years, yet few published studies have addressed why and how older people have been involved as partners in research. This article presents a systematic review of the literature, examining the aims and challenges of co-research and the ways in which older adults can be involved in research. Systematic searches covering several databases were conducted, yielding a total of 3,293 articles, with 27 papers reviewed which involved older people as co-researchers across more than one stage of the research cycle. We find that co-research with older people offers improved understandings of the issues facing older people; more inclusive and responsive policy, practice and service design; and opportunities for co-researchers to develop new skills whilst giving voice to marginalised groups of older people. The analysis highlights the different ways in which older adults can be involved across phases of research, and identifies ethical, methodological and practical challenges encountered in the process. Involving older adults as co-researchers, while labour intensive and practically challenging, holds promise as an underdeveloped resource for social gerontology, as well as older people themselves. The paper identifies four pathways for improving and extending co-research: developing diversified structures of involvement, supporting co-researchers, embedding research rigour and ensuring co-ownership of change.
\end{abstract}

Keywords: literature review; participatory research; co-production; co-research; ageing; older people

\section{Introduction}

The ageing of the population, together with the need for more inclusive and responsive policies and services, has contributed to a growing interest in co-production and co-research with older people. 'Co-research' in this article is defined as doing research 'with' or 'by' older adults rather than 'to', 'about' or 'for' them (Fudge et al., 2007). It is used as an umbrella term to encompass a family of approaches, such as 'participatory', 'emancipatory' and 'inclusive' research,

(c) The Author(s), 2022. Published by Cambridge University Press. This is an Open Access article, distributed under the terms of the Creative Commons Attribution licence (http://creativecommons.org/licenses/by/4.0/), which permits unrestricted re-use, distribution and reproduction, provided the original article is properly cited. 
which reflect a turn towards involving communities in the process of knowledge production. Co-research aims to put principles of empowerment into practice, by offering participants greater control over the research process while providing opportunities to learn and reflect upon their experience (Durose et al., 2011). It challenges both the asymmetry of power relations between researchers and researched, and the invisibility of older adults in terms of their lived experiences and diversity (Ray, 2007). Co-research has, at its root, a commitment to challenging the dominant discourse which tends to construct ageing as an individualised 'problem' with a focus on illness and decline (Ziegler and Scharf, 2013; Hyde et al., 2014). As such, co-research represents a value-based approach to doing research which espouses the importance of diversity and a commitment to valuing older people's perspectives and lived experiences, together with a critical exploration of the structural factors that lead to exclusion, oppression and marginalisation in later life. Co-research seeks to involve older adults fully in the research process and has the potential to shift established power imbalances and contribute to social action to improve the quality of life in later life (Buffel, 2018).

While the co-research approach has existed since the 1980s, e.g. in the work of Averil Osborn (1985; see also Osborn and Willcocks, 1990), several factors have recently stimulated interest in the idea of co-production and creating opportunities for older people to become active partners in research. These include: first, pressure for more personalised and responsive services (Blair and Minkler, 2009); second, the growth of self-advocacy movements with different groups advocating their right to be active participants in research and policy design (Walker, 2007); and third, policies coming under the banner of 'active ageing' (in Europe) and 'successful ageing' (in the United States of America (USA)) (Pruchno, 2015; World Health Organization, 2015) emphasising the value of participation and engagement in later life. Co-production approaches further align with the current focus on 'user engagement' and 'research impact' emphasised by funding bodies and policy organisations (Buffel et al., 2017), offering the potential for research 'to deliver significant societal impact via dynamic, locally adaptive community-academic partnerships' (Greenhalgh et al., 2016: 392).

Although research in partnership with older people has been slower to develop compared with other groups, a growing body of work suggests that co-research may help to understand some of the complex health and social problems experienced by older people, at the same time as promoting individual and community capacity building (Ward and Barnes, 2016). In their literature review of participatory action research in gerontology, Blair and Minkler (2009: 651) argue that co-research 'helps ensure that the topic under investigation matters locally; improves the relevance and cultural sensitivity of ... data collection tools; adds nuance to the interpretation of findings and [assists] in the translation of findings into action'. Other benefits associated with this approach include its potential for promoting the empowerment of marginalised groups of older people and improving the quality of community and health services drawing on the perspective of its users (Fudge et al., 2007).

Despite its potential, the 'participatory turn' in ageing research has not gone unchallenged. Littlechild et al. (2015) found that the most common forms of engagement are skewed towards a 'tokenistic approach' in which older people have little influence over the research process, keeping them at the lower end of 
the participation ladder because of professional assumptions about the place of participants in research (Arnstein, 1969; Ray, 2007). This has been further established in a recent critical interpretive review which found that older adults are very rarely involved as prominent or equitable partners in participatory research (Corrado et al., 2020), a tendency reinforced by the time-consuming and under-funded nature of this type of work (Walker, 2007). However, only a limited amount of published work is available showing whether and how co-research with older people can be practically realised (Littlechild et al., 2015). Moreover, there is increasing recognition of the challenges faced in involving more vulnerable groups within the older population, challenges which can exacerbate power differentials within and between groups (Buffel, 2018). Learning from existing projects and critically evaluating the state of the art of older people's participation in research is therefore essential (Ray, 2007), especially if the aim is to 'promote genuine and high-level involvement of older adults that respects their needs and concerns; honors and builds on their strengths; and, in the process, helps address power imbalances' (Blair and Minkler, 2009: 658). A reflexive approach, meaning one which engages in critical reflection about the role of researchers and co-researchers in the production of knowledge represents, offers an important means to advance knowledge about co-research with older people.

Against this background, this article provides a systematic review of empirical studies that have involved older adults as co-researchers. The paper addresses three research questions:

(1) What are the benefits of involving older people in co-produced research?

(2) In what ways have older people been involved in different phases of the research cycle?

(3) What are the challenges associated with involving older people in research?

The studies examined in this review will highlight several core themes and issues that need to be addressed if co-research with older adults is to reach its full potential.

\section{Design and methods}

A systematic literature review was conducted to ensure a comprehensive and unbiased search and assessment of studies involving older people as co-researchers (Heyn et al., 2019). The review used Preferred Reporting Items for Systematic Reviews and Meta-Analyses (PRISMA) (Moher et al., 2009; Heyn et al., 2019), as reported in the supplementary material. Both authors were involved in screening, applying the eligibility criteria and selecting studies for inclusion in the review, with the search strategy reviewed by a specialist from the University of Manchester library.

\section{Search strategy and study selection}

The search and selection strategy consisted of three steps: (a) searching for relevant studies, (b) screening records and (c) applying inclusion criteria. The first step involved searching for publications that involved older people as active participants in the design 
Table 1. Search platforms and databases

\begin{tabular}{ll}
\hline Search platform & \multicolumn{1}{c}{ Databases } \\
\hline EBSCO & $\begin{array}{l}\text { Abstracts in Social Gerontology, CINHAHL Complete, ERIC, } \\
\text { Humanities Full Text, Humanities Source, MEDLINE, PsycARTICLES, } \\
\text { Psychology and Behavioural Sciences Collection, PsycINFO, Social } \\
\text { Sciences Full Text, Social Work Abstracts, SocINDEX Full Text } \\
\text { databases }\end{array}$ \\
\hline Ovid & YourJournals@Ovid, OvidMedline \\
Web of Science & SSCI, A\&HCIS \\
Scopus & Scopus proprietary \\
\hline
\end{tabular}

and conduct of the research. The search was conducted across four platforms to cover multiple disciplines, research fields and countries, as detailed in Table 1.

The search terms were divided into two themes: older adult (using the terms older adults/people and elderly) and co-research methods (using the terms participatory/action/participatory action research, co-research and co-production). The two sets of terms were combined with the Boolean operator AND, meaning that a record had to include at least one of the first set and at least one of the second set of terms in order to be identified. The search terms were applied on title, abstract and the full text. We did not use age-based criteria (or age bands which were built into some of the search platforms) to allow for variations in ageing across different groups. The search was limited to English-language records within the last 20 years (1999-2019). In total, 3,257 records were identified through database searching.

The second step involved the screening of the 3,257 records to narrow down the number of papers to be considered for review. At this stage, we excluded 3,100 papers which did not specifically involve older people in research, including studies which described the involvement of older people in other activities, such as health promotion activities or personal care plans (Fudge et al., 2007). A database was created in Excel to facilitate the screening of titles, keywords and abstracts. A proportion $(10 \%)$ of records were independently assessed by both researchers to improve the reliability of the process. As agreement between the reviewers on ex/included papers was high (94\%), and because of the large number of papers involved, the remainder of the records were reviewed by the first author, with unclear results discussed by both reviewers until consensus was reached. To supplement the database search, we contacted experts in the field of co-research with older people to identify forthcoming publications that met the inclusion criteria. Thirty-six studies were brought to our attention. Finally, duplicate records were removed to ensure a valid and reliable pool of studies for inclusion in the review (Figure 1).

The third step involved the assessment of 161 full-text articles to determine whether they met the key criterion to be included in the review, i.e. the active involvement of older adults, following the definition of doing research 'with' or 'by' rather than 'to', 'about' or 'for' older people (Fudge et al., 2007). For the purpose of this study, we defined 'active' to mean involvement of older adults across 


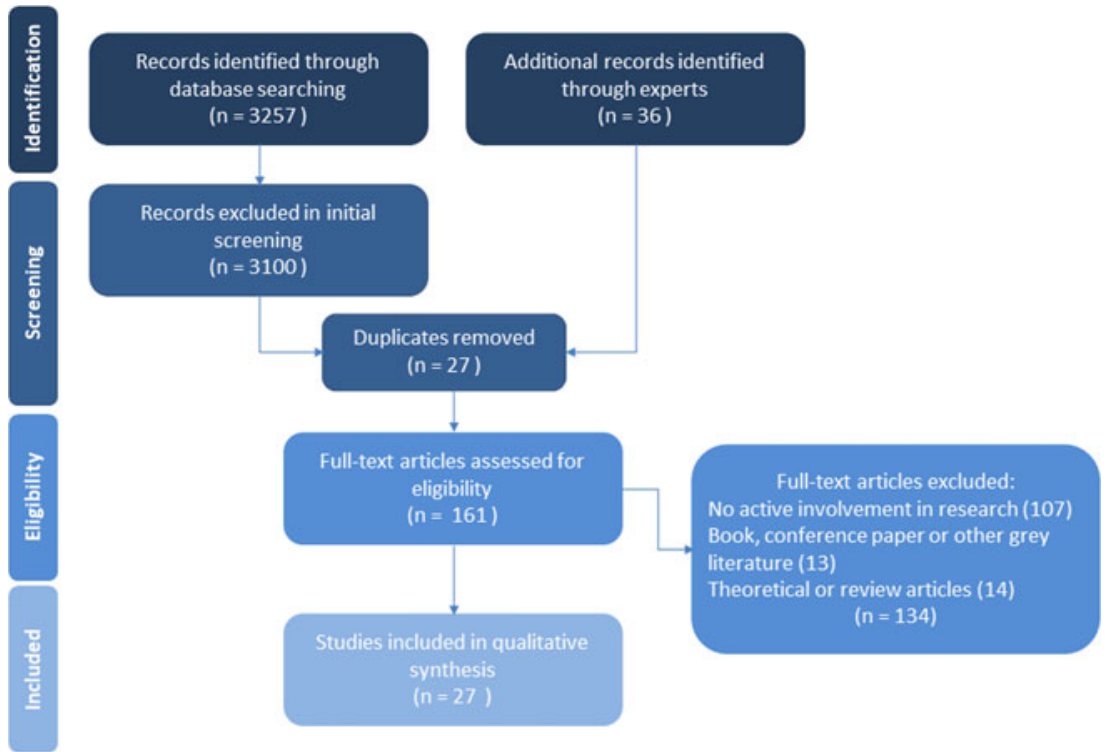

Figure 1. Flow of information through the review (based on Moher et al., 2009).

more than one stage of the research cycle (i.e. developing the study, designing the study, collecting and analysing data, and disseminating findings), in order to examine the most detailed cases of genuine involvement in co-research. ${ }^{1}$ In addition, we only included publications that were peer-reviewed, as an accepted indication of quality; and thus, excluded grey literature, book chapters, conference papers, dissertations and policy documents. The application of inclusion criteria was led by the first author and reviewed by the second author, with disagreements (such as whether the paper met our definition of active involvement) discussed until consensus was reached. This resulted in 27 unique articles being identified for review (Figure 1).

\section{Extraction and data analysis}

Following PRISMA (Moher et al., 2009), a structured data extraction form was created in Excel to record the following categories: research objectives, design, methodology, participants, results and outcomes. We then critically assessed the quality of the studies, using the criteria of validity (i.e. appropriateness and accuracy of the design, methodology, tools and processes used), reflexivity (sensitivity to the ways in which the (co)researcher(s) and research process had shaped the collected data), relevance (whether the study added to existing knowledge) and the quality of reporting of study design and conduct (Mays and Pope, 2000). The data extraction form was pre-tested by both reviewers collecting data from five studies to assess the validity of the form. A refined version was then used to analyse all articles, with the first author taking the lead in synthesising findings for each of the categories, and the second author checking and validating results. Differences in individual judgements were highlighted and discussed until consensus was reached. 


\section{Findings}

Table 2 shows the 27 papers, with brief summaries of the research setting, co-researchers, nature of co-researcher involvement, methods used and outcomes. Some papers concern the same research project, indicated in the table, or otherwise involve the same researchers. The majority of studies (20) were based in Europe: 16 in the United Kingdom (UK), two in Ireland, and one each in the Netherlands and Belgium. There were four studies in the USA, one in Canada, one in Israel and one in Tanzania. Most of the studies (22) were community or neighbourhood based, with 17 in urban areas, four in rural areas and one including both area types. The other five studies were based on specific services or projects which were not geographically bound. In summary, the studies covered co-researchers aged from mid-fifties to early eighties, with some studies involving a broad age range within one project.

\section{The benefits of involving older people as co-researchers}

This section addresses the first research question regarding the benefits of involving older people as co-investigators in research. Four categories were identified: first, achieving a deeper understanding and improved data; second, developing the skills and experiences of co-researchers; third, creating change in policy, services, practice and the community; and fourth, challenging power dynamics. These categories were not mutually exclusive, with many studies reporting them together.

\section{Achieving a deeper understanding and improved data}

The first category concerned developing a deeper understanding of the experiences of older adults and the issues to be considered in research by including older people in designing and developing the study (e.g. Doyle and Timonen, 2010; Tanner, 2012; Brown et al., 2017; Mey and van Hoven, 2019). Research conducted 'from the perspective of those immediately concerned by the issues being investigated, rather than through the lens of researchers' preconceptions and priorities' (Doyle and Timonen, 2010: 245) was linked to enhanced data quality for three reasons.

First, older co-researchers are able to converse and empathise with interviewees, who feel more at ease to share their perspective with a peer-researcher, leading to richer data (Mey and van Hoven, 2019). For example, in a study involving co-researchers with dementia, Tanner (2012: 299) reported that 'the shared identity of being someone with dementia would facilitate relationships between researchers and participants, thus enhancing the experience of the interview process for both parties, as well as enriching the data obtained.'

Second, the shared experiences between older co-researchers and marginalised groups of older people who participated in the research also meant that researchers were better able to recognise what to ask or pay attention to when issues were raised. In a participatory study aimed at developing age-friendly communities in low-income neighbourhoods in Manchester, UK, co-researchers highlighted 'the benefits of sharing certain characteristics with interviewees (e.g., age, local knowledge, ethnicity) for engaging with those experiencing isolation and from minority ethnic backgrounds' (Buffel, 2019: 546; see also Buffel, 2018). 
Table 2. Summary of studies reviewed

\begin{tabular}{|c|c|c|c|c|c|c|c|}
\hline No. & Source & Research setting & Aims & Co-researchers & Involvement & Methods & Outcomes \\
\hline 1 & $\begin{array}{l}\text { Barnes } \\
\text { et al. } \\
\text { (2013) }\end{array}$ & $\begin{array}{l}\text { City in south-east } \\
\text { England, in } \\
\text { partnership with } \\
\text { local } \\
\text { voluntary-sector } \\
\text { organisation }\end{array}$ & $\begin{array}{l}\text { Involving older } \\
\text { people as } \\
\text { co-producers of } \\
\text { knowledge to } \\
\text { improve } \\
\text { understandings of } \\
\text { wellbeing }\end{array}$ & $\begin{array}{l}12 \text { older adults } \\
\text { aged early } 60 \mathrm{~s} \text { to } \\
\text { late } 80 \mathrm{~s} \text {, recruited } \\
\text { through partner } \\
\text { organisation }\end{array}$ & $\begin{array}{l}\text { Research } \\
\text { design, data } \\
\text { collection, } \\
\text { analysis }\end{array}$ & $\begin{array}{l}\text { Qualitative } \\
\text { interviews: } \\
\text { co-researchers } \\
\text { conducted } 30 \\
\text { interviews, coded } \\
\text { interview data and } \\
\text { analysed themes } \\
\text { collectively }\end{array}$ & $\begin{array}{l}\text { Strengthened } \\
\text { findings around } \\
\text { the relational and } \\
\text { generative nature } \\
\text { of wellbeing }\end{array}$ \\
\hline 2 & $\begin{array}{l}\text { Bishop } \\
\text { et al. } \\
\text { (2013) }\end{array}$ & $\begin{array}{l}\text { Small } \\
\text { south-central city } \\
\text { in the USA }\end{array}$ & $\begin{array}{l}\text { Understanding } \\
\text { perspective of older } \\
\text { people faced with } \\
\text { environmental } \\
\text { inequities. Amplify } \\
\text { voices of } \\
\text { marginalised } \\
\text { individuals }\end{array}$ & $\begin{array}{l}1 \text { participant aged } \\
70 \text { years, who was } \\
\text { a local resident } \\
\text { living with } \\
\text { disabilities }\end{array}$ & $\begin{array}{l}\text { Data } \\
\text { collection, } \\
\text { analysis, } \\
\text { dissemination }\end{array}$ & $\begin{array}{l}\text { Photovoice: } \\
\text { co-researcher took } \\
\text { pictures, analysed } \\
\text { them thematically } \\
\text { and produced } \\
\text { materials for } \\
\text { dissemination }\end{array}$ & $\begin{array}{l}\text { Raised awareness } \\
\text { amongst the } \\
\text { community and } \\
\text { led to local } \\
\text { changes }\end{array}$ \\
\hline 3 & $\begin{array}{l}\text { Brown } \\
\text { et al. } \\
(2017)\end{array}$ & $\begin{array}{l}\text { Volunteer } \\
\text { programme in a } \\
\text { community in } \\
\text { mid-west USA, } \\
\text { supported by } \\
\text { community } \\
\text { partner } \\
\text { organisations }\end{array}$ & $\begin{array}{l}\text { Understanding the } \\
\text { impact of the } \\
\text { volunteer } \\
\text { programme on } \\
\text { wellbeing, health } \\
\text { and quality of life, } \\
\text { to improve } \\
\text { experiences }\end{array}$ & $\begin{array}{l}60 \text { adults aged } 55 \\
\text { years and over, } \\
\text { who participated } \\
\text { in the volunteer } \\
\text { programme }\end{array}$ & $\begin{array}{l}\text { Research } \\
\text { design, data } \\
\text { collection }\end{array}$ & $\begin{array}{l}\text { Surveys: } \\
\text { co-researchers } \\
\text { designed survey to } \\
\text { measure wellbeing. } \\
\text { Focus groups: } \\
\text { co-researchers } \\
\text { participated in } \\
\text { discussion-based } \\
\text { focus groups }\end{array}$ & $\begin{array}{l}\text { Led to greater } \\
\text { collaboration and } \\
\text { understanding } \\
\text { between partners }\end{array}$ \\
\hline 4 & $\begin{array}{l}\text { Buffel } \\
\text { (2019) }\end{array}$ & $\begin{array}{l}\text { Three communities } \\
\text { in a city in } \\
\text { north-west UK }\end{array}$ & $\begin{array}{l}\text { Exploring } \\
\text { age-friendly } \\
\text { communities } \\
\text { through an insider } \\
\text { perspective. Making }\end{array}$ & $\begin{array}{l}18 \text { older adults } \\
\text { aged } 58-74 \text { years, } \\
\text { reflecting the } \\
\text { ethnic and social }\end{array}$ & $\begin{array}{l}\text { Research } \\
\text { design, } \\
\text { recruitment, } \\
\text { data collection, }\end{array}$ & $\begin{array}{l}\text { Qualitative } \\
\text { interviews: } \\
\text { co-researchers } \\
\text { designed the study, } \\
\text { conducted }\end{array}$ & $\begin{array}{l}\text { Demonstrated } \\
\text { that older people } \\
\text { brought skills to } \\
\text { the project. } \\
\text { Informed policy }\end{array}$ \\
\hline
\end{tabular}


Table 2. (Continued.)

\begin{tabular}{|c|c|c|c|c|c|c|c|}
\hline No. & Source & Research setting & Aims & Co-researchers & Involvement & Methods & Outcomes \\
\hline & & & $\begin{array}{l}\text { older people } \\
\text { central to policy } \\
\text { and practice }\end{array}$ & $\begin{array}{l}\text { make-up of the } \\
\text { communities }\end{array}$ & $\begin{array}{l}\text { analysis, } \\
\text { dissemination }\end{array}$ & $\begin{array}{l}\text { qualitative } \\
\text { interviews, analysed } \\
\text { data thematically }\end{array}$ & $\begin{array}{l}\text { change in } \\
\text { community }\end{array}$ \\
\hline 5 & $\begin{array}{l}\text { Buffel } \\
(2018)\end{array}$ & $\begin{array}{l}\text { Three communities } \\
\text { in a city in } \\
\text { north-west UK }\end{array}$ & $\begin{array}{l}\text { Exploring } \\
\text { age-friendly } \\
\text { communities } \\
\text { through an insider } \\
\text { perspective }\end{array}$ & $\begin{array}{l}18 \text { older adults } \\
\text { aged } 58-74 \text { years, } \\
\text { reflecting the } \\
\text { ethnic and social } \\
\text { make-up of the } \\
\text { communities }\end{array}$ & $\begin{array}{l}\text { Research } \\
\text { design, } \\
\text { recruitment, } \\
\text { data collection, } \\
\text { analysis, } \\
\text { dissemination }\end{array}$ & $\begin{array}{l}\text { Qualitative } \\
\text { interviews: } \\
\text { co-researchers } \\
\text { designed the study, } \\
\text { conducted } \\
\text { qualitative interviews } \\
\text { and analysed data } \\
\text { thematically }\end{array}$ & $\begin{array}{l}\text { Demonstrated } \\
\text { benefits of } \\
\text { co-research for } \\
\text { older people } \\
\text { involved, } \\
\text { especially } \\
\text { seldom-heard } \\
\text { groups }\end{array}$ \\
\hline 6 & $\begin{array}{l}\text { Doran and } \\
\text { Buffel } \\
(2018)\end{array}$ & $\begin{array}{l}\text { Three communities } \\
\text { in a city in } \\
\text { north-west UK }\end{array}$ & $\begin{array}{l}\text { Making older } \\
\text { people central to } \\
\text { development of } \\
\text { evidence-based } \\
\text { policy and practice. } \\
\text { Including } \\
\text { marginalised voices }\end{array}$ & $\begin{array}{l}18 \text { older adults } \\
\text { aged } 58-74 \text { years, } \\
\text { with maximum } \\
\text { variation to reflect } \\
\text { ethnic and social } \\
\text { diversity }\end{array}$ & $\begin{array}{l}\text { Research } \\
\text { design, } \\
\text { recruitment, } \\
\text { data collection, } \\
\text { analysis, } \\
\text { dissemination }\end{array}$ & $\begin{array}{l}\text { Qualitative } \\
\text { interviews: } \\
\text { co-researchers } \\
\text { organised and } \\
\text { participated in } \\
\text { stakeholder meetings } \\
\text { and public } \\
\text { engagement events }\end{array}$ & $\begin{array}{l}\text { Informed policy } \\
\text { change in } \\
\text { community and } \\
\text { wider region }\end{array}$ \\
\hline 7 & $\begin{array}{l}\text { Doyle and } \\
\text { Timonen } \\
(2010)\end{array}$ & $\begin{array}{l}\text { Based in the } \\
\text { community of a } \\
\text { suburb of Dublin, } \\
\text { Ireland, working } \\
\text { with stakeholders }\end{array}$ & $\begin{array}{l}\text { Understanding } \\
\text { needs of older } \\
\text { people in local } \\
\text { community. } \\
\text { Breaking down } \\
\text { power } \\
\text { distributions, } \\
\text { empowering } \\
\text { participants }\end{array}$ & $\begin{array}{l}26 \text { older adults in } \\
\text { total, all resident } \\
\text { in geographic } \\
\text { boundary of the } \\
\text { study }\end{array}$ & $\begin{array}{l}\text { Research } \\
\text { design, } \\
\text { recruitment, } \\
\text { data collection, } \\
\text { analysis }\end{array}$ & $\begin{array}{l}\text { Questionnaire: } \\
\text { co-researchers } \\
\text { recruited } \\
\text { participants, } \\
\text { administered } \\
\text { questionnaires, } \\
\text { commented on data } \\
\text { analysis and the final } \\
\text { report }\end{array}$ & $\begin{array}{l}\text { Roll-out of new } \\
\text { community } \\
\text { initiatives. } \\
\text { Involvement of } \\
\text { older } \\
\text { co-researchers } \\
\text { strengthened } \\
\text { findings }\end{array}$ \\
\hline 8 & $\begin{array}{l}\text { Ellins and } \\
\text { Glasby } \\
(2016)\end{array}$ & $\begin{array}{l}\text { Case study site in a } \\
\text { City Council and a }\end{array}$ & $\begin{array}{l}\text { Developing deeper } \\
\text { insight into the } \\
\text { hospital }\end{array}$ & $\begin{array}{l}8 \text { older adults } \\
\text { from minority }\end{array}$ & $\begin{array}{l}\text { Research } \\
\text { design, data }\end{array}$ & $\begin{array}{l}\text { Qualitative } \\
\text { interviews: } \\
\text { co-researchers }\end{array}$ & $\begin{array}{l}\text { Findings highlight } \\
\text { specific } \\
\text { challenges for }\end{array}$ \\
\hline
\end{tabular}




\begin{tabular}{|c|c|c|c|c|c|c|c|}
\hline & & $\begin{array}{l}\text { wider Shire County } \\
\text { in England, UK }\end{array}$ & $\begin{array}{l}\text { experiences of } \\
\text { older people from } \\
\text { minority ethnic } \\
\text { communities; } \\
\text { empowering } \\
\text { co-researchers }\end{array}$ & $\begin{array}{l}\text { ethnic } \\
\text { communities }\end{array}$ & $\begin{array}{l}\text { collection, } \\
\text { analysis }\end{array}$ & $\begin{array}{l}\text { conducted } \\
\text { qualitative research } \\
\text { interviews with a } \\
\text { researcher and } \\
\text { participated in } \\
\text { analysis workshops }\end{array}$ & $\begin{array}{l}\text { minority groups. } \\
\text { Improved } \\
\text { knowledge of } \\
\text { these issues }\end{array}$ \\
\hline 9 & $\begin{array}{l}\text { Fenge } \\
(2010)\end{array}$ & $\begin{array}{l}\text { Local initiative in } \\
\text { city in south UK, in } \\
\text { partnership with } \\
\text { local voluntary } \\
\text { agency }\end{array}$ & $\begin{array}{l}\text { Understanding } \\
\text { experiences of } \\
\text { sexuality and } \\
\text { ageing. Supporting } \\
\text { marginalised } \\
\text { groups to inform } \\
\text { local agencies } \\
\text { about their needs }\end{array}$ & $\begin{array}{l}\text { Older lesbians } \\
\text { and gay men } \\
\text { living and } \\
\text { volunteering in } \\
\text { local area }\end{array}$ & $\begin{array}{l}\text { Research } \\
\text { design, data } \\
\text { collection, } \\
\text { analysis, } \\
\text { dissemination }\end{array}$ & $\begin{array}{l}\text { Qualitative interviews } \\
\text { and questionnaires: } \\
\text { co-researchers were } \\
\text { involved in designing, } \\
\text { conducting, } \\
\text { analysing and writing } \\
\text { the report }\end{array}$ & $\begin{array}{l}\text { Demonstrates } \\
\text { insider knowledge } \\
\text { and 'variant } \\
\text { truths' gained } \\
\text { through } \\
\text { co-researcher } \\
\text { involvement }\end{array}$ \\
\hline 10 & $\begin{array}{l}\text { Fenge } \\
\text { et al. } \\
(2009)\end{array}$ & $\begin{array}{l}\text { Local initiative in } \\
\text { city in south UK, in } \\
\text { partnership with } \\
\text { local voluntary } \\
\text { agency }\end{array}$ & $\begin{array}{l}\text { Understanding } \\
\text { experiences of } \\
\text { sexuality and } \\
\text { ageing. Supporting } \\
\text { marginalised } \\
\text { groups to inform } \\
\text { local agencies } \\
\text { about their needs }\end{array}$ & $\begin{array}{l}\text { Older lesbians } \\
\text { and gay men } \\
\text { living and } \\
\text { volunteering in } \\
\text { local area }\end{array}$ & $\begin{array}{l}\text { Research } \\
\text { design, data } \\
\text { collection, } \\
\text { analysis, } \\
\text { dissemination }\end{array}$ & $\begin{array}{l}\text { Qualitative interviews } \\
\text { and questionnaires: } \\
\text { co-researchers were } \\
\text { involved in designing, } \\
\text { conducting, } \\
\text { analysing and writing } \\
\text { the report }\end{array}$ & $\begin{array}{l}\text { The project was } \\
\text { transformative on } \\
\text { personal and } \\
\text { societal levels }\end{array}$ \\
\hline 11 & $\begin{array}{l}\text { Fenge } \\
\text { et al. } \\
(2010)\end{array}$ & $\begin{array}{l}\text { Rural area in south } \\
\text { England, working } \\
\text { with a local service } \\
\text { provider }\end{array}$ & $\begin{array}{l}\text { Understanding } \\
\text { experiences of } \\
\text { older lesbian and } \\
\text { gay citizens in rural } \\
\text { areas. Giving voice } \\
\text { to and empowering } \\
\text { marginalised } \\
\text { groups }\end{array}$ & $\begin{array}{l}\text { Older lesbians } \\
\text { and gay men } \\
\text { living in the area, } \\
\text { recruited through } \\
\text { snowball } \\
\text { sampling }\end{array}$ & $\begin{array}{l}\text { Research } \\
\text { design, data } \\
\text { collection, } \\
\text { analysis, } \\
\text { dissemination }\end{array}$ & $\begin{array}{l}\text { Mixed methods: } \\
\text { co-researchers were } \\
\text { involved in } \\
\text { biographical } \\
\text { narrative interpretive } \\
\text { interviews, visual } \\
\text { ethnography, focus } \\
\text { groups and theatrical } \\
\text { workshops }\end{array}$ & $\begin{array}{l}\text { Empowered } \\
\text { marginalised } \\
\text { voices. Raised } \\
\text { awareness and } \\
\text { changed } \\
\text { perceptions }\end{array}$ \\
\hline 12 & $\begin{array}{l}\text { Gutman } \\
\text { et al. } \\
\text { (2014) }\end{array}$ & $\begin{array}{l}\text { College in Tel Aviv, } \\
\text { Israel, in } \\
\text { collaboration with }\end{array}$ & $\begin{array}{l}\text { Involving older } \\
\text { adults as } \\
\text { co-researchers for }\end{array}$ & $\begin{array}{l}6 \text { older service } \\
\text { users aged } 72-80 \\
\text { years, active }\end{array}$ & $\begin{array}{l}\text { Data } \\
\text { collection, } \\
\text { analysis }\end{array}$ & $\begin{array}{l}\text { Mixed methods: } \\
\text { co-researchers were } \\
\text { involved in }\end{array}$ & $\begin{array}{l}\text { Findings helped } \\
\text { inform future } \\
\text { courses at the }\end{array}$ \\
\hline
\end{tabular}


Table 2. (Continued.)

\begin{tabular}{|c|c|c|c|c|c|c|c|}
\hline No. & Source & Research setting & Aims & Co-researchers & Involvement & Methods & Outcomes \\
\hline & & $\begin{array}{l}\text { UK academic } \\
\text { institution }\end{array}$ & $\begin{array}{l}\text { richer and more } \\
\text { relevant findings to } \\
\text { inform social work } \\
\text { education }\end{array}$ & $\begin{array}{l}\text { members of local } \\
\text { community; } 5 \\
\text { women, } 1 \text { man }\end{array}$ & & $\begin{array}{l}\text { interviewing } \\
\text { participants, } \\
\text { analysing } \\
\text { questionnaires, } \\
\text { vignettes and } \\
\text { concept maps }\end{array}$ & $\begin{array}{l}\text { college. Personal } \\
\text { benefits to older } \\
\text { people involved }\end{array}$ \\
\hline 13 & $\begin{array}{l}\text { Hand et al. } \\
\text { (2019) }\end{array}$ & $\begin{array}{l}\text { Based in a seniors' } \\
\text { apartment } \\
\text { building in a } \\
\text { Canadian city }\end{array}$ & $\begin{array}{l}\text { Promoting social } \\
\text { connectedness and } \\
\text { inclusion amongst } \\
\text { residents }\end{array}$ & $\begin{array}{l}15 \text { residents aged } \\
50 \text { years and over }\end{array}$ & $\begin{array}{l}\text { Research } \\
\text { design, data } \\
\text { collection }\end{array}$ & $\begin{array}{l}\text { Mixed methods: } \\
\text { co-researchers were } \\
\text { collaboratively } \\
\text { involved in designing } \\
\text { interventions and } \\
\text { surveys across the } \\
\text { project }\end{array}$ & $\begin{array}{l}\text { Demonstrates } \\
\text { insights into the } \\
\text { participatory } \\
\text { action research } \\
\text { process and how } \\
\text { to work } \\
\text { collaboratively }\end{array}$ \\
\hline 14 & $\begin{array}{l}\text { Jones } \\
\text { et al. } \\
\text { (2013) }\end{array}$ & $\begin{array}{l}\text { Rural area in south } \\
\text { England, working } \\
\text { with a local service } \\
\text { provider }\end{array}$ & $\begin{array}{l}\text { Understanding } \\
\text { experiences of } \\
\text { older lesbian and } \\
\text { gay citizens in rural } \\
\text { areas. Giving voice } \\
\text { to and empowering } \\
\text { marginalised } \\
\text { groups }\end{array}$ & $\begin{array}{l}\text { Older lesbians } \\
\text { and gay men } \\
\text { living in the area, } \\
\text { recruited through } \\
\text { snowball } \\
\text { sampling }\end{array}$ & $\begin{array}{l}\text { Research } \\
\text { design, data } \\
\text { collection, } \\
\text { analysis, } \\
\text { dissemination }\end{array}$ & $\begin{array}{l}\text { Mixed methods: } \\
\text { co-researchers were } \\
\text { involved in } \\
\text { biographical } \\
\text { narrative interpretive } \\
\text { interviews, visual } \\
\text { ethnography, focus } \\
\text { groups and theatrical } \\
\text { workshops }\end{array}$ & $\begin{array}{l}\text { Promoted insider } \\
\text { knowledge } \\
\text { through } \\
\text { biographic } \\
\text { methods }\end{array}$ \\
\hline 15 & $\begin{array}{l}\text { Littlechild } \\
\text { et al. } \\
\text { (2015) }\end{array}$ & $\begin{array}{l}\text { Four study sites in } \\
\text { the UK, based on } \\
\text { local dementia } \\
\text { service provision }\end{array}$ & $\begin{array}{l}\text { Understanding the } \\
\text { experiences of } \\
\text { older people, } \\
\text { including } \\
\text { marginalised } \\
\text { groups in research. } \\
\text { Evaluating impact }\end{array}$ & $\begin{array}{l}22 \text { co-researchers } \\
\text { across four sites }\end{array}$ & $\begin{array}{l}\text { Research } \\
\text { design, data } \\
\text { collection, } \\
\text { analysis, } \\
\text { evaluation }\end{array}$ & $\begin{array}{l}\text { Qualitative } \\
\text { interviews: } \\
\text { co-researchers were } \\
\text { involved in designing } \\
\text { interview tools, } \\
\text { conducted } 75 \\
\text { interviews, thematic } \\
\text { data analysis and } \\
\text { evaluation }\end{array}$ & $\begin{array}{l}\text { Demonstrated a } \\
\text { shift in power } \\
\text { dynamics of } \\
\text { research. } \\
\text { Empowered } \\
\text { participants }\end{array}$ \\
\hline
\end{tabular}




\begin{tabular}{|c|c|c|c|c|c|c|c|}
\hline 16 & $\begin{array}{l}\text { Mey and } \\
\text { van Hoven } \\
(2019)\end{array}$ & $\begin{array}{l}\text { City } \\
\text { neighbourhood in } \\
\text { north Netherlands }\end{array}$ & $\begin{array}{l}\text { Understanding } \\
\text { perspectives of } \\
\text { older people about } \\
\text { age-friendly cities. } \\
\text { Giving voice to and } \\
\text { empowering older } \\
\text { people to influence } \\
\text { change }\end{array}$ & $\begin{array}{l}6 \text { older adults } \\
\text { aged } 49-82 \text { years; } \\
1 \text { woman, } 5 \text { men }\end{array}$ & $\begin{array}{l}\text { Research } \\
\text { design, data } \\
\text { collection, } \\
\text { analysis, } \\
\text { dissemination }\end{array}$ & $\begin{array}{l}\text { Qualitative } \\
\text { interviews: } \\
\text { co-researchers were } \\
\text { involved in the } \\
\text { research design, } \\
\text { conducted six } \\
\text { qualitative interviews } \\
\text { each, analysed data } \\
\text { and led } \\
\text { dissemination }\end{array}$ & $\begin{array}{l}\text { Co-researchers } \\
\text { felt empowered } \\
\text { as they acted as } \\
\text { advocates }\end{array}$ \\
\hline 17 & $\begin{array}{l}\text { Pan et al. } \\
(2019)\end{array}$ & $\begin{array}{l}\text { Region with } 59 \\
\text { municipalities in } \\
\text { Belgium }\end{array}$ & $\begin{array}{l}\text { Exploring the role } \\
\text { of media usage } \\
\text { amongst older } \\
\text { people in Belgium, } \\
\text { as integral part of } \\
\text { healthy ageing }\end{array}$ & $\begin{array}{l}30-100 \text { volunteers } \\
\text { per municipality, } \\
\text { managed by a } \\
\text { volunteer } \\
\text { supervisor }\end{array}$ & $\begin{array}{l}\text { Recruitment, } \\
\text { data collection }\end{array}$ & $\begin{array}{l}\text { Questionnaire: } \\
\text { co-researchers } \\
\text { contacted selected } \\
\text { participants, } \\
\text { supported } \\
\text { completion, and } \\
\text { returned data for } \\
\text { collation and analysis }\end{array}$ & $\begin{array}{l}\text { Older people } \\
\text { found to be an } \\
\text { essential partner } \\
\text { in the research }\end{array}$ \\
\hline 18 & $\begin{array}{l}\text { Porter } \\
(2016)\end{array}$ & $\begin{array}{l}\text { Ten settlements in } \\
\text { rural Tanzania }\end{array}$ & $\begin{array}{l}\text { Understanding } \\
\text { mobility constraints } \\
\text { faced by older } \\
\text { people. Addressing } \\
\text { power balance in } \\
\text { research }\end{array}$ & $\begin{array}{l}12 \text { men and } \\
\text { women aged 59- } \\
69 \text { years, living in } \\
\text { local settlements }\end{array}$ & $\begin{array}{l}\text { Research } \\
\text { design, data } \\
\text { collection, } \\
\text { dissemination }\end{array}$ & $\begin{array}{l}\text { Qualitative } \\
\text { interviews: } \\
\text { co-researchers } \\
\text { trialled research } \\
\text { method then } \\
\text { conducted } \\
\text { interviews; } \\
\text { co-researchers } \\
\text { presented findings at } \\
\text { a national workshop }\end{array}$ & $\begin{array}{l}\text { Co-researchers } \\
\text { had improved } \\
\text { confidence and } \\
\text { reported findings } \\
\text { to stakeholders }\end{array}$ \\
\hline 19 & $\begin{array}{l}\text { Pratesi } \\
\text { et al. } \\
\text { (2013) }\end{array}$ & $\begin{array}{l}\text { UK-based local } \\
\text { project supported } \\
\text { by Technology } \\
\text { Strategy Board and } \\
\text { industrial partner }\end{array}$ & $\begin{array}{l}\text { Using } \\
\text { people-centred } \\
\text { processes to lead } \\
\text { to better design. } \\
\text { Highlighting the } \\
\text { importance of }\end{array}$ & $\begin{array}{l}6 \text { adults from } \\
\text { different cultural, } \\
\text { social and } \\
\text { occupational } \\
\text { backgrounds }\end{array}$ & $\begin{array}{l}\text { Research } \\
\text { design, data } \\
\text { collection, } \\
\text { analysis }\end{array}$ & $\begin{array}{l}\text { Mixed methods: } \\
\text { co-researchers fed } \\
\text { into research design } \\
\text { and into analysis; } \\
\text { co-researchers were } \\
\text { involved in data }\end{array}$ & $\begin{array}{l}\text { Deeper insight } \\
\text { gained from } \\
\text { involvement of } \\
\text { older people. } \\
\text { Personal benefits } \\
\text { for co-researchers }\end{array}$ \\
\hline
\end{tabular}


Table 2. (Continued.)

\begin{tabular}{|c|c|c|c|c|c|c|c|}
\hline No. & Source & Research setting & Aims & Co-researchers & Involvement & Methods & Outcomes \\
\hline & & & $\begin{array}{l}\text { participatory } \\
\text { research } \\
\text { approaches }\end{array}$ & & & $\begin{array}{l}\text { collection, through } \\
\text { interviews, focus } \\
\text { groups, trials and } \\
\text { secondary data } \\
\text { analysis }\end{array}$ & \\
\hline 20 & $\begin{array}{l}\text { Shore } \\
\text { et al. } \\
(2018)\end{array}$ & $\begin{array}{l}\text { City in mid-west } \\
\text { Ireland }\end{array}$ & $\begin{array}{l}\text { Understanding the } \\
\text { needs of older } \\
\text { people to develop } \\
\text { age-friendly } \\
\text { designs. Raising } \\
\text { awareness that } \\
\text { participatory } \\
\text { design leads to } \\
\text { better solutions }\end{array}$ & $\begin{array}{l}22 \text { older adults, } \\
\text { aged } 69-80 \text {, living } \\
\text { independently in } \\
\text { the local } \\
\text { community }\end{array}$ & $\begin{array}{l}\text { Data gathering, } \\
\text { analysis, } \\
\text { dissemination }\end{array}$ & $\begin{array}{l}\text { Mixed methods: } \\
\text { co-researchers were } \\
\text { involved in cultural } \\
\text { probe activities and } \\
\text { participated in a } \\
\text { design symposium to } \\
\text { analyse data and } \\
\text { disseminate to key } \\
\text { stakeholders }\end{array}$ & $\begin{array}{l}\text { Demonstrated the } \\
\text { benefits of } \\
\text { collaboration to } \\
\text { achieve efficient } \\
\text { and tangible } \\
\text { solutions }\end{array}$ \\
\hline 21 & $\begin{array}{l}\text { Tanner } \\
(2012)\end{array}$ & $\begin{array}{l}\text { Case study on local } \\
\text { dementia services } \\
\text { in the UK, part of a } \\
\text { national study with } \\
\text { local partner } \\
\text { agency }\end{array}$ & $\begin{array}{l}\text { Understanding } \\
\text { older people's } \\
\text { experiences of } \\
\text { dementia services. } \\
\text { Enhancing } \\
\text { interview data and } \\
\text { improving } \\
\text { confidence of } \\
\text { co-researchers }\end{array}$ & $\begin{array}{l}3 \text { co-researchers } \\
\text { aged } 60,71 \text { and } \\
77 \text {, living with } \\
\text { dementia; } 1 \\
\text { woman, } 2 \text { men. } \\
\text { Recruited through } \\
\text { partner } \\
\text { organisation }\end{array}$ & $\begin{array}{l}\text { Data gathering, } \\
\text { analysis }\end{array}$ & $\begin{array}{l}\text { Qualitative } \\
\text { interviews: } \\
\text { co-researchers } \\
\text { conducted interviews } \\
\text { individually and } \\
\text { thematic analysis } \\
\text { collectively, with lead } \\
\text { researcher } \\
\text { supporting }\end{array}$ & $\begin{array}{l}\text { Involvement } \\
\text { meant better } \\
\text { relationships } \\
\text { which enriched } \\
\text { the interviews, } \\
\text { data and findings. } \\
\text { Personal benefits } \\
\text { for co-researchers }\end{array}$ \\
\hline 22 & $\begin{array}{l}\text { Ward } \\
(2014)\end{array}$ & $\begin{array}{l}\text { City in south UK, } \\
\text { working with local } \\
\text { voluntary-sector } \\
\text { organisation }\end{array}$ & $\begin{array}{l}\text { Investigating older } \\
\text { people's narratives } \\
\text { of relationships and } \\
\text { relationality, how } \\
\text { these relate to } \\
\text { wellbeing. Involving } \\
\text { older people as } \\
\text { co-producers of } \\
\text { knowledge }\end{array}$ & $\begin{array}{l}12 \text { older adults } \\
\text { aged early } 60 \text { s to } \\
\text { late } 80 \text { s, recruited } \\
\text { through partner } \\
\text { organisation }\end{array}$ & $\begin{array}{l}\text { Research } \\
\text { design, data } \\
\text { gathering, } \\
\text { analysis }\end{array}$ & $\begin{array}{l}\text { Qualitative } \\
\text { interviews: } \\
\text { co-researchers } \\
\text { conducted } 30 \\
\text { interviews, coded } \\
\text { interview data and } \\
\text { discussed themes } \\
\text { collectively }\end{array}$ & $\begin{array}{l}\text { Strengthened } \\
\text { findings around } \\
\text { the relational and } \\
\text { generative nature } \\
\text { of wellbeing }\end{array}$ \\
\hline
\end{tabular}




\begin{tabular}{|c|c|c|c|c|c|c|c|}
\hline 23 & $\begin{array}{l}\text { Ward and } \\
\text { Barnes } \\
(2016)\end{array}$ & $\begin{array}{l}\text { City in south-east } \\
\text { England in } \\
\text { partnership with } \\
\text { local } \\
\text { voluntary-sector } \\
\text { organisation }\end{array}$ & $\begin{array}{l}\text { Involving older } \\
\text { people as } \\
\text { co-producers of } \\
\text { knowledge, } \\
\text { improve } \\
\text { understandings of } \\
\text { wellbeing }\end{array}$ & $\begin{array}{l}12 \text { older adults } \\
\text { aged early } 60 \text { s to } \\
\text { late } 80 \text { s, recruited } \\
\text { through partner } \\
\text { organisation }\end{array}$ & $\begin{array}{l}\text { Research } \\
\text { design, data } \\
\text { gathering, } \\
\text { analysis, } \\
\text { dissemination }\end{array}$ & $\begin{array}{l}\text { Co-researchers were } \\
\text { involved in data } \\
\text { analysis and } \\
\text { dissemination with } \\
\text { key stakeholders, } \\
\text { developing learning } \\
\text { resources for working } \\
\text { with older people }\end{array}$ & $\begin{array}{l}\text { Demonstrated the } \\
\text { need for a } \\
\text { framework to } \\
\text { involve older } \\
\text { people in social } \\
\text { work practice }\end{array}$ \\
\hline 24 & $\begin{array}{l}\text { Ward et al. } \\
(2011)\end{array}$ & $\begin{array}{l}\text { City in south UK, } \\
\text { working with local } \\
\text { agencies }\end{array}$ & $\begin{array}{l}\text { Investigating older } \\
\text { people's } \\
\text { experiences of } \\
\text { alcohol use in later } \\
\text { life. Achieving } \\
\text { deeper insight by } \\
\text { engaging older } \\
\text { co-researchers }\end{array}$ & $\begin{array}{l}4 \text { older } \\
\text { co-researchers, } \\
\text { aged mid-50s to } \\
60 \mathrm{~s} ; 3 \text { women, } 1 \\
\text { man; } 5 \text { older } \\
\text { people were } \\
\text { members of } \\
\text { reference group }\end{array}$ & $\begin{array}{l}\text { Recruitment, } \\
\text { research } \\
\text { design, data } \\
\text { collection, } \\
\text { analysis }\end{array}$ & $\begin{array}{l}\text { Qualitative } \\
\text { interviews: } \\
\text { co-researchers } \\
\text { recruited } \\
\text { participants, } \\
\text { commented on topic } \\
\text { guide, conducted } \\
\text { qualitative interviews } \\
\text { and analysed data } \\
\text { thematically }\end{array}$ & $\begin{array}{l}\text { Novel findings } \\
\text { around alcohol } \\
\text { use in later life } \\
\text { and the need for } \\
\text { engagement of } \\
\text { older people in } \\
\text { research }\end{array}$ \\
\hline 25 & $\begin{array}{l}\text { Ward and } \\
\text { Gahagan } \\
(2010)\end{array}$ & $\begin{array}{l}\text { City in south UK, } \\
\text { working with local } \\
\text { voluntary-sector } \\
\text { organisation }\end{array}$ & $\begin{array}{l}\text { Developing a } \\
\text { person-centred } \\
\text { approach to } \\
\text { research with older } \\
\text { people. } \\
\text { Understanding } \\
\text { wellbeing for older } \\
\text { people. Breaking } \\
\text { down power } \\
\text { barriers }\end{array}$ & $\begin{array}{l}\text { People aged } 60- \\
87 \text { years recruited } \\
\text { through the } \\
\text { partner } \\
\text { organisation }\end{array}$ & $\begin{array}{l}\text { Research } \\
\text { design, data } \\
\text { gathering, } \\
\text { analysis }\end{array}$ & $\begin{array}{l}\text { Qualitative interviews } \\
\text { and focus groups: } \\
\text { co-researchers } \\
\text { conducted } \\
\text { qualitative interviews } \\
\text { and focus groups; } \\
\text { co-researchers coded } \\
\text { interview data and } \\
\text { advised on findings }\end{array}$ & $\begin{array}{l}\text { Empowered older } \\
\text { people who were } \\
\text { involved. } \\
\text { Collaboration was } \\
\text { beneficial to } \\
\text { knowledge }\end{array}$ \\
\hline 26 & $\begin{array}{l}\text { Winter } \\
\text { et al. } \\
(2016)\end{array}$ & $\begin{array}{l}\text { Communities in } \\
\text { east USA, high } \\
\text { proportion of } \\
\text { Latino population }\end{array}$ & $\begin{array}{l}\text { Gathering, } \\
\text { analysing and } \\
\text { disseminating } \\
\text { information about } \\
\text { features that affect } \\
\text { active living. } \\
\text { Reaching }\end{array}$ & $\begin{array}{l}10 \text { older adults } \\
\text { with mean age } \\
71.3 \pm 6.5 \text { years, } \\
\text { all female, } \\
\text { recruited through } \\
\text { local community } \\
\text { organisations }\end{array}$ & $\begin{array}{l}\text { Data gathering, } \\
\text { analysis, } \\
\text { dissemination }\end{array}$ & $\begin{array}{l}\text { Environmental } \\
\text { assessments: } \\
\text { co-researchers used } \\
\text { the Stanford Healthy } \\
\text { Neighborhood } \\
\text { Discovery Tool to } \\
\text { collect } 366 \text { photos }\end{array}$ & $\begin{array}{l}\text { Identified } \\
\text { overlooked issues } \\
\text { and led to } \\
\text { positive change in } \\
\text { the local area. } \\
\text { Co-researchers } \\
\text { gained skills and }\end{array}$ \\
\hline
\end{tabular}


Table 2. (Continued.)

\begin{tabular}{|c|c|c|c|c|c|c|c|}
\hline No. & Source & Research setting & Aims & Co-researchers & Involvement & Methods & Outcomes \\
\hline & & & $\begin{array}{l}\text { marginalised } \\
\text { groups to } \\
\text { understand barriers }\end{array}$ & & & $\begin{array}{l}\text { and narratives; } \\
\text { co-researchers } \\
\text { analysed data and } \\
\text { presented findings to } \\
\text { stakeholders }\end{array}$ & $\begin{array}{l}\text { improved } \\
\text { self-efficacy }\end{array}$ \\
\hline 27 & $\begin{array}{l}\text { Yankeelov } \\
\text { et al. } \\
\text { (2015) }\end{array}$ & $\begin{array}{l}\text { Three rural } \\
\text { communities in the } \\
\text { USA, partnership } \\
\text { with regional } \\
\text { planning agency } \\
\text { coalition }\end{array}$ & $\begin{array}{l}\text { Understanding } \\
\text { experiences of } \\
\text { older adults living } \\
\text { with diabetes in } \\
\text { rural communities; } \\
\text { promoting dialogue } \\
\text { and knowledge; } \\
\text { advocating for } \\
\text { policy change }\end{array}$ & $\begin{array}{l}23 \text { older adult } \\
\text { co-researchers } \\
\text { aged } 60-78 \text { years } \\
\text { living with Type } 2 \\
\text { diabetes; } 18 \\
\text { women, } 5 \text { men }\end{array}$ & $\begin{array}{l}\text { Data } \\
\text { collection, } \\
\text { analysis, } \\
\text { dissemination }\end{array}$ & $\begin{array}{l}\text { Photovoice: } \\
\text { co-researchers took } \\
\text { photos of their } \\
\text { personal } \\
\text { environment and } \\
\text { community, analysed } \\
\text { the images } \\
\text { thematically } \\
\text { individually and in } \\
\text { group }\end{array}$ & $\begin{array}{l}\text { Findings led to } \\
\text { increased } \\
\text { collective } \\
\text { awareness, policy } \\
\text { change and } \\
\text { co-researcher } \\
\text { empowerment }\end{array}$ \\
\hline
\end{tabular}

Notes: UK: United Kingdom. USA: United States of America. 
Finally, a deeper understanding of the experiences of older people from ethnic minority older groups was 'powerfully illustrated by the accounts of participants who were interviewed by co-researchers in [shared] community languages, showing the value that participatory approaches can have for accessing and understanding the experiences of groups that have been largely marginalised from research' (Ellins and Glasby, 2016: 58).

\section{Developing the skills and experiences of co-researchers}

The second category was linked to the opportunities for co-researchers to gain new experiences, build relationships and networks, and develop new skills, such as conducting interviews, engaging with stakeholder groups, and fostering collective advocacy and community change (e.g. Tanner, 2012; Gutman et al., 2014; Winter et al., 2016; Buffel, 2018; Hand et al., 2019). For example, Winter et al. explored how neighbourhood features affect active living amongst disadvantaged communities in the USA and reported that:

A community engaged approach was used in which participants were provided with advocacy and leadership training, which equipped them to engage with local policy makers to initiate neighborhood improvements relevant to healthy active living. (Winter et al., 2016: 1127)

Whilst some studies reported such benefits as an aim of the research, many others had not fully anticipated the social and personal gains for co-researchers at the start of the project, reporting these as an outcome instead. For example, Tanner (2012) found that co-researchers with dementia benefited from sharing experiences and exchanging coping strategies with other co-researchers and participants, facilitating the development of mutually supportive relationships. Gutman et al. (2014: 192), in a study of experiences of social work education among students from a local college, illustrated how the research reinforced participants' self-worth using a quote from one of the co-researchers: 'I suddenly realised they were talking about me! I was that old person who gave the student that understanding about what old age is all about.'

\section{Creating change in policy, services, practice and the community}

The third category concerned creating change in policy, services, practice and the community to address the marginalised position of older people in society through direct changes to structures which affect their everyday lives. Examples included studies aimed at improving health services for people with dementia (Tanner, 2012), developing neighbourhoods to support active living for disadvantaged groups (Winter et al., 2016) and promoting the participation of marginalised groups of older adults in policy making (Yankeelov et al., 2015). In a study using photovoice methods with older adults with diabetes in rural America, Yankeelov et al. reported:

Photovoice is used to promote critical dialogue and knowledge about important issues ... to advocate for change ... This method provides participants, who traditionally have little voice in community policy decisions, with training in 
photography, critical dialog, photo captioning, and policy advocacy. (Yankeelov et al., 2015: 202)

One concrete outcome from this study included a sidewalk that was repaired as a result of discussions between co-researchers and policy makers about road transport barriers on the way to public exercise facilities (Yankeelov et al., 2015). Other projects illustrated how research findings were translated into community resource guides (Winter et al., 2016) and strategic action plans (Buffel, 2018) with initiatives to improve the social and physical environment for older people.

\section{Challenging power dynamics}

The final category involved challenging power dynamics, referring to societal structures of inequality and injustice related to ageing which marginalise older people (Fenge, 2010; Fenge et al., 2010; Jones et al., 2013). By providing opportunities for confidence building, skills development, training and social action, co-research projects have the potential to empower older people to speak out against discrimination and oppression, and for change in systems (Ray, 2007). This was illustrated in a study by Fenge et al. (2010) in which lesbian and gay older people produced a film about experiences of ageing in rural Britain. The film was used to raise public awareness, encourage community dialogue and inform service providers, but also provided an opportunity for older people to express their sexual identity whilst highlighting intersecting exclusionary pressures linked to homophobia, heterosexism and age. Another example came from Littlechild et al. (2015) who found that co-researchers with dementia felt that the study had helped them to own and affirm publicly their dementia identity in a way that benefited others, challenging negative expectations of both self and others. Ward and Gahagan (2010) further illustrated how co-research can challenge power arrangements by breaking down traditional knowledge hierarchies about whose knowledge is valued above others, highlighting the value of involving older people in the process of knowledge production.

\section{Methods of involving older people in co-research}

This section addresses the second research question: in what ways have older people been involved in research? The analysis highlighted one key theme that cut across the various phases of research, i.e. the role of training. This will be discussed first, followed by older people's participation in developing and designing the study, collecting and analysing data, and disseminating findings.

\section{Training to support co-researcher's involvement}

Training formed a critical part of most co-research projects, with the organisation of multiple training sessions at the start of each research phase being the most common approach to avoid time lags between learning and doing. Training was often structured around key topics reflecting the different phases in research, and delivered in a workshop format, allowing for interaction, reflection and practice (Porter, 2016; Mey and van Hoven, 2019). In some cases, the training was designed so that it was especially sensitive to more marginalised voices in the community. In a study 
on age-friendly neighbourhoods, for example, issues such as minimising cultural biases, sensitivity to racial and ethnic diversity, and asymmetric power relationships formed part of the topics discussed with the co-researchers in the workshops (Buffel, 2019).

\section{Developing the study}

This first phase refers to the activities to set up a research project, including developing the research problem, generating support, acquiring ethical approval and forming a project team. Very few studies reported how older co-researchers were involved in this stage: those that did emphasised the role of partnerships and trust building between agencies, academics and older people early in the project (e.g. Fenge et al., 2010; Yankeelov et al., 2015; Brown et al., 2017). In Yankeelov et al. (2015), a funded coalition consisting of service providers, charities and older residents was set up to oversee the project and ensure its focus on tackling health disparities for rural people with diabetes. Similarly, in a study on experiences of sexuality and ageing, co-researchers were involved in developing the study through 'an Advisory Committee made up of a mix of older gay people and service providers who are central in the development and overview of the project' (Fenge et al., 2010: 323-324). In both examples, the research advisory committee provided an infrastructure for members to voice concerns and priorities that otherwise might not have entered the research agenda.

\section{Designing the study}

While the involvement of older adults in developing the study was rather limited, most studies provided insights into how co-researchers contributed to the design of the project, including deciding on various aspects of the methodology, such as the approach to recruitment and data collection, and the selection of research tools (e.g. Ward et al., 2011, Ward, 2014; Buffel, 2019; Hand et al., 2019). For example, in a study addressing alcohol use in later life by Ward et al. (2011: 241), co-researchers were involved in determining how best to approach recruitment given the sensitive nature of the topic. They decided on the project title 'Cheers!? A project about older people and alcohol' and selected images to be used on a flyer which was successfully used to recruit participants. Older co-researchers also significantly influenced the design of a study on wellbeing in later life (Ward, 2014) by opting for semi-structured interviews and focus groups rather than the originally planned survey methods to explore the role of social relationships in the experience of wellbeing in later life.

\section{Collecting data}

All studies had involved co-researchers in data collection, and there were three methods for doing so. The first, most common, method involved co-researchers collecting data from other people as research subjects, such as interviewing older people about hospital experiences (Ellins and Glasby, 2016), interviewing students about experiences of social work education (Gutman et al., 2014) or using questionnaires to examine social participation amongst older adults (Pan et al., 2019). In these instances, co-researchers often used their networks to recruit potential participants into the study. Second, in some studies, the co-researcher became the subject of research, using their own experiences to address research questions (Bishop 
et al., 2013; Yankeelov et al., 2015; Winter et al., 2016). For example, in a study about independent living, Linda, a dually disabled 70-year-old woman, used photovoice methods to explore and articulate her own experiences in navigating her home and local area (Bishop et al., 2013). Being in charge of the data collection offered Linda control over issues of self-representation, which may be particularly important to marginalised groups of older people. Finally, a third group of studies used a variety of methods, involving older people as both researchers and research subjects (Fenge et al., 2010; Pratesi et al., 2013; Shore et al., 2018). These projects often included creative data collection methods and interactive outputs for dissemination. For instance, in Fenge et al. (2010), co-researchers were involved in biographical narrative interpretive interviews, visual ethnography, focus groups and theatrical improvisation workshops, which led to the creation of a film reflecting the findings of the research.

\section{Analysing data}

Almost all reviewed studies suggested that older adults had been involved in analysing data, highlighting the benefits associated with 'co-researchers reflect[ing] on the data from their own lived experience, enabling a richer understanding of the meaning and significance of interviewees' responses' (Barnes et al., 2013: 483). However, it was often unclear how this had occurred, with a particular lack of reporting detail around the approach to analysing data, the tools used to support this process and the role that co-researchers played. The few studies that did report their approach most commonly used a thematic coding framework, which tended to be most effective when used collaboratively, for three reasons: first, co-researchers reported difficulties in working alone whilst collaborative ways of analysing data were found to lead to richer findings (Gutman et al., 2014); second, independent coding entails the risk for different transcripts to have different foci, creating issues in bringing the analysis together (Mey and van Hoven, 2019); and third, analysing data in a collaborative way reduces the risk of individual (co)researchers focusing too narrowly on the data and dismissing or overlooking issues (Buffel, 2019).

The analysis identified different approaches to involving co-researchers in writing up research findings. Only one paper included a co-researcher as an author (Bishop et al., 2013), however, some studies referenced other outputs that were jointly authored by the co-researchers, such as project reports or other grey literature. Additionally, some studies included co-researchers' voice through the use of reflections and feedback collected over the course of the project. For example, the papers by Fenge et al. $(2009,2010)$ focused on narrative accounts of the experiences of co-researchers gathered at the end of the project which illustrated the transformative nature of their involvement in the research. Finally, some studies reported that co-researchers were invited to review and comment on the written outputs that were produced by academic researchers (e.g. Doyle and Timonen, 2010; Pratesi et al., 2013).

\section{Disseminating findings}

Many studies reported that co-researchers were involved in disseminating research findings, e.g. through participating in public engagement events and workshops, 
producing resources for partner organisations or presenting at public conferences (e.g. Yankeelov et al., 2015; Doran and Buffel, 2018; Shore et al., 2018). These studies illustrated that older co-researchers have an important advocacy role in disseminating findings to key stakeholders. For example, Yankeelov et al. (2015) found that recommendations suggested by co-researchers, such as promoting healthier food options and investing in local support groups, had been acted upon following their engagement in community stakeholder events. This study also raised the importance of advocacy training to support older people involved in dissemination, which was echoed in other projects.

\section{The unfolding challenges}

This section addresses the third research question regarding the challenges associated with co-research with older people. The analysis identified three key themes: first, power differentials and the risk of reproducing inequalities; second, challenges associated with the different expectations, roles and forms of participation; and third, issues around sustainability and resources.

\section{Power differentials and the risk of reproducing inequalities}

Many co-research studies were aimed at addressing prevalent power differentials by meaningfully involving older people in research. However, whilst seeking to democratise knowledge production and fostering opportunities for those involved, co-research nevertheless constitutes a form of social power. Co-researchers have opportunity to influence societal knowledge and practices through the research project, which carries the risk of reproducing the inequalities that it seeks to address (Buffel, 2018). For example, Porter (2016), in her study in rural Tanzania, reflected on how co-research may create a further divide between an already more 'privileged' group of older people and their more disadvantaged peers, contributing to forms of 'disempowerment' for more excluded groups, through the emphasis placed on the differences in power, capabilities and expertise between co-researchers and their interviewees:

The studies reported here involved small, arguably already privileged groups in peer research - albeit also relatively vulnerable by virtue of their poverty ... or age ... the peer-researchers already had the privilege of some formal education ... This then poses the question; can members of these favored groups adequately engage with and re-present all their age cohort? (Porter, 2016: 300)

Another manifestation of power differentials concerns payments to co-researchers. Remuneration may be essential to encourage and enable participation from people who would not be likely to be involved otherwise, yet very few studies discussed their approach to paying co-researchers. Where it was mentioned, projects tended to offer out-of-pocket expenses and/or compensation to co-researchers. For example, in Yankeelov et al. (2015), a study involving older adults with diabetes in rural America, co-researchers were compensated for their time and effort across the project to the sum of US \$225 per person. In contrast, in Gutman et al. (2014), co-researchers were offered payment for their work, but declined (although expenses were covered). 


\section{Different expectations, roles and forms of involvement}

A second challenge was linked to co-researchers resisting the participatory nature of the study. While this review focused on studies which involved co-researchers across multiple phases of the research, active involvement was not always what older people wanted or were able to commit to. Bishop et al. (2013: 302-303), for example, reported that the co-researcher in their project 'seemed to recreate the traditional dichotomous research situation of expert/layperson inherent in positivist approaches to inquiry'. Other studies observed similar issues among partner organisations who had more outcome-driven expectations from the research. For instance, in the Smart Monitoring project, Pratesi et al. (2013: 10) reported difficulties among expert technologists in accepting and adopting the participatory and iterative nature of the design process. This questions assumptions regarding what can be considered genuine involvement, and highlights that navigating and negotiating differing expectations, roles and forms of involvement, which may change and evolve over the course of the project, is a critical feature of any co-research project (Walker, 2007).

\section{Issues around sustainability, time and resources}

Finally, the analysis suggested that sustainability is a significant issue and challenge, in part due to the resource-intensive nature of this type of work. Most studies engaged with multiple actors at different stages, to take into account their needs and concerns throughout the process, to carefully encourage, recruit, support and train co-researchers, as well as involve them in collecting data and sharing and implementing findings. This implies the need for sufficient resources, not only in terms of research funding, but also in terms of human support, energy and commitment. Yet this is not always recognised by funding agencies (Walker, 2007; Corrado et al., 2020). Doyle and Timonen echo this point:

The time-intensiveness of the process needs to be appreciated by policy makers and funding agencies that propound the advantages of [participatory research] but are in many cases not prepared to invest the funds required to permit an extended working relationship between the community and researchers. (Doyle and Timonen, 2010: 256)

Studies that involved older people in developing age-friendly neighbourhoods further suggested that co-research methods are most appropriate when there are opportunities for long-term engagement to address issues and develop actions (Buffel, 2019; Mey and van Hoven, 2019). However, the potential for immediate social and political change may be limited in the context of a short-term research project with limited funding. This was found to be a source of frustration for older participants, especially those who have had a lifetime of disadvantage and deprivation.

\section{Discussion}

Interest in co-research with older adults has grown in the past 20 years (Dewar, 2005; Ross et al., 2005; Clough et al., 2006; Boyce et al., 2009), and this article contributes to the research agenda by providing a systematic review of empirical studies 
that have actively involved older adults as co-researchers. It contributes new insights into the outcomes of co-research, and the processes by which this method can promote a better understanding of the variety of social and health issues facing older people whilst contributing to individual and community capacity building (Ross et al., 2005; Blair and Minkler, 2009; Durose et al., 2011).

The value of co-research is at least threefold: first, it can play a vital role in challenging negative stereotypes of ageing by emphasising and developing the skills and knowledge which older people can bring to research. Second, it provides opportunities for partnerships between older people, service providers and community stakeholders working together to stimulate change in policy, services and practice to improve quality of life in later life. Third, it provides a method for challenging traditional power arrangements and ensuring voice and visibility of marginalised groups, with the potential of empowering older people to speak out against discrimination and oppression, and for change in systems. The paper further reveals the range of methodological approaches that can be adopted to involve older adults meaningfully across multiple phases of the research, demonstrating how involvement can be tailored for different methods, topics and contexts, making the potential opportunities for co-research evident.

Despite opportunities, the review also highlights challenges linked to the co-research approach, notably those associated with managing conflicting expectations and responsibilities; barriers to achieving sustainability; and the challenge of developing collaborative partnerships and negotiating power relationships between the different groups involved. In terms of the latter, there is a risk that projects which recruit and train older people to become co-researchers further empower those who already have considerable social capital while adding to the exclusion of more marginalised groups. This raises the possibility that co-research creates a further divide between an already privileged group of older people and their more disadvantaged peers. Without recognition of such tensions, co-research may reinforce rather than reduce existing inequalities between groups (Littlechild et al., 2015; Porter, 2016; Buffel, 2019; Bendien et al., in press).

The studies examined in this review also provide insights into how some of these challenges can be addressed whilst promoting the authentic engagement of diverse groups of older people in research. Several core themes and lessons emerged that need to be further addressed if co-research with older adults is to reach its full potential. These include: first, developing diversified structures of involvement; second, supporting co-researchers; third, embedding research rigour; and fourth, ensuring co-ownership of change.

\section{Developing diversified structures of involvement}

This review highlights the importance of designing the involvement in research in a way that accommodates the lived experiences of older people. This suggests the need for understanding older adults' motivations for, and expectations from, being involved as co-researchers to offer potential roles and responsibilities which suit their needs (Dewar, 2005; Ray, 2007; Blair and Minkler, 2009; Bendien et al., in press). A good practice in this respect was found in the study by Pratesi et al. (2013: 8), which used terms of reference to provide a flexible framework and enable co-researchers to determine their own involvement. Similarly, 
other scholars suggest that co-research projects should offer a range of flexibly adaptable roles and responsibilities, to reflect the various, and potentially changing, ways that older people may want to be involved over the course of a project (Ray, 2007; Rémillard-Boilard et al., 2017). A diversification of co-researcher roles must include considering the needs of less-privileged groups and developing structures which accommodate and reflect the diversity of ageing experiences in relation to gender, ethnicity, class and sexuality. It should also recognise that the needs of co-researchers may change during the project, and that this requires potential routes to increase, lessen or stop their involvement at different stages of the project.

\section{Supporting co-researchers}

The studies included in this review underline the value of, and need for, training and supporting older adults in their role as co-researchers, both in terms of improving data collection and analysis, as well as developing participant's skills, confidence and self-esteem. In line with the need for diversified structures of involvement (see above), the analysis suggests that co-researchers benefit significantly from personalised forms of support and mentoring. One way to achieve this involves the creation of spaces which enhance peer-to-peer support and learning, and where co-researchers can reflect upon and share their feelings with other co-researchers as well as the academic researchers. Investing in building trust between different members of the team and fostering a culture of openness, mutual support and co-learning are necessary to facilitate this.

To support this, some co-research studies adopt an 'ethics of care' framework to developing ethical and mutually supportive relationships within the research team as well as with older persons who participate in the study. Such a framework is based upon interconnected principles such as attentiveness, responsibility, competence, responsiveness and trust (Ward and Gahagan, 2010; Ward and Barnes, 2016). Involving older people as co-researchers, then, is not just a matter of including them in a set of research activities, it also concerns the shaping of the nature of the relationships developed during the process. This involves being attentive to the different contributions that co-researchers feel comfortable with making, as well as to the practical support that is necessary to enable co-learning and co-research.

\section{Embedding principles for improving the rigour of co-research}

The analysis further shows that there is room for co-research to gain traction by embedding principles which improve the rigour of participatory methodologies (Blair and Minkler, 2009; Corrado et al., 2020; Bendien et al., in press). Following a critical assessment of the studies according to key criteria of research quality (Mays and Pope, 2000), two areas for improving rigour in co-research can be identified. The first concerns the need for more reflexivity, especially regarding the issue of who the co-researchers are and how they may have shaped the research. Few projects reported the details of recruitment or characteristics of the co-researchers, whether this changed over the course of the project (i.e. attrition), and if so the reasons why this was the case. There was little reflection on how these aspects could have shaped the data collected and the inclusivity of the project. Similarly, some projects involved a very broad age range of co-researchers, with 
little consideration of what this means for the understanding of later life generated. One recommendation for future co-research projects is therefore to foster reflection and discussion about the implications of the co-researchers' positionality, and their ways of knowing and constructing meaning, for the research process. This is especially important in relation to power and privilege differentials that may exist between older co-researchers and research participants or interviewees (Bishop et al., 2013; Littlechild et al., 2015).

The second area concerns validity, which refers to the appropriateness and accuracy of the research methodology. Most studies included in this review did not fully describe how co-researchers were involved in different phases of the research, nor did they include the co-researcher's perspective on their involvement (Blair and Minkler, 2009). While the absence of such details may be linked to limitations associated with publishing in academic journals, reporting the details of the co-researchers' involvement, including their perspective, would not only improve the quality of the study itself but also contribute to the need for sharing good practice. Some studies demonstrate the importance of incorporating the voice of the co-researcher in the project write-up, e.g. through the inclusion of direct quotes or involving co-researchers as co-authors, which gives greater strength to the claims made about their experiences (McKillop and Wilkinson, 2004; Fenge et al., 2009; Gutman et al., 2014; Buffel, 2019).

\section{Co-ownership of change}

This review has demonstrated the ways in which co-research can contribute to achieving change, e.g. through promoting policy interventions, creating new advocacy groups or changing attitudes about older people. This was most powerful where co-researchers were able to co-create and take ownership of such change (Barnes, 2005; Ray, 2007; Blair and Minkler, 2009; Corrado et al., 2020). This was evident in research on age-friendly communities in Manchester, UK, where co-researchers instigated a campaign to reinstate a local bus service which led to the formation of a permanent group advocating for the needs of older residents (Doran and Buffel, 2018; Buffel, 2019). Similarly, co-researchers involved in a study to identify barriers to active living in the USA formed a new local steering committee aimed at tackling illegal dumping, an issue highlighted by the research (Winter et al., 2016).

The analysis has identified three pathways through which co-ownership of change can be promoted. The first concerns involving co-researchers from the start of the project to develop a shared understanding of what the research aims to achieve. The review shows that a limited number of studies involve older people in the first phase of the research cycle, in developing the study and defining the research aims and aspired outcomes. There is, thus, considerable scope to foster co-ownership by working in partnership with older people and other stakeholders in shaping the research agenda. Second, there is also a need for involving co-researchers in evaluating the impact of the participatory approach, both in terms of their own experience as well as the project outcomes. Where studies had included the voice of the co-researchers, it added to the validity of the study as well as generating directions for future co-research projects (McKillop and 
Wilkinson, 2004; Hyde et al., 2014). Finally, there were many examples where co-researchers continued to advocate for change based on the study findings after the project ended. Supporting initiatives initiated by the co-researchers and jointly developing opportunities for future co-research projects may further promote a sense of co-ownership.

\section{Limitations of the review}

There are two limitations associated with this review. First, we only identified studies that specifically used the terms older adults/people or elderly. There could be projects where older people are involved, without reference to their age status, which may represent even greater diversity in the ways in which older people can be involved in co-research. Second, our study is further limited by restricting our searches to peer-reviewed journals and English-language papers. The grey literature, including books and reports, and papers in other languages, may provide additional information about co-research involving older adults.

\section{Conclusion}

In their literature review on participatory action research for The Gerontologist over a decade ago, Blair and Minkler argued that:

the time appears ripe for ... expanding social and critical gerontology's scope to include research with rather than solely on older adults and their caregivers. In so doing, we may expand, as well, the relevance of our field for studying and addressing the complex health and social problems faced by elders but also these individuals' unique strengths and the invaluable knowledge they can offer as coresearchers. (Blair and Minkler, 2009: 661)

Echoing this theme, Walker (2007: 482) has suggested that 'Access to the meaning of old age cannot legitimately be attempted scientifically without the active engagement of older people as participants of various kinds in the research process rather than as "objects".

This article has reviewed the literature on co-research with older adults, providing a response to the need for expanding methodological diversity and innovation in ageing research while incorporating older people as participants into the process of research and theorising. It examines why and how older adults have been 'actively' involved in co-research, defined in this paper as participation in more than one stage of the research cycle, moving away from tokenistic and consumerist approaches to participation, to emphasise the importance of collective action and citizen rights (Ray, 2007). With increased diversity and inequality in ageing populations globally, it is essential to treat older people as active citizens in the processes of knowledge creation and change which impact their lives and the communities in which they live (Marsh, 1981; Blair and Minkler, 2009). The paper offers four pathways for improving and expanding the use of co-research methods in gerontology, including: developing diversified structures of involvement, supporting co-researchers, embedding rigour and enhancing co-ownership of change. 
Supplementary material. The supplementary material for this article can be found at https://doi.org/10. $1017 /$ S0144686X21002014

Author contributions. All authors have made a substantial contribution to the conception and design, or analysis and interpretation of data, and the drafting and approval of this article.

Financial support. This work was supported by the Economic and Social Research Council (ESRC) under the Future Research Leaders scheme (grant number ES/N002180/1); and the Leverhulme Trust (RL-2019-011).

Conflict of interest. The authors declare no conflicts of interest.

Ethical standards. This project did not collect empirical data so ethical approval was not required.

\section{Note}

1 We acknowledge that it is possible to involve older adults meaningfully and actively in only one phase of the research and that involvement in two or more phases can also be tokenistic, yet our aim was to examine the most detailed and/or involved examples of co-research.

\section{References}

Arnstein SR (1969) A ladder of citizen participation. Journal of the American Institute of Planners 35, 216224.

Barnes M (2005) The same old process? Older people, participation and deliberation. Ageing \& Society 25, 245-259.

Barnes M, Taylor D and Ward L (2013) Being well enough in old age. Critical Social Policy 33, 473-493.

Bendien E, Groot B and Abma T (in press) Circles of impacts within and beyond participatory action research with older people. Ageing \& Society. Available online doi:10.1017/S0144686X20001336.

Bishop J, Robillard L and Moxley D (2013) Linda's story through photovoice: achieving independent living with dignity and ingenuity in the face of environmental inequities. Practice 25, 297-315.

Blair T and Minkler M (2009) Participatory action research with older adults: key principles in practice. The Gerontologist 49, 651-662.

Boyce M, O'Brien N, Munn-Giddings C and McVicar A (2009) How does the rhetoric of 'user participation' in research apply to older people? Research, Policy and Planning 27, 55-63.

Brown ER, Lu Y, Beaven J, Hendrie HC, Hanson K, Carvell CA and Austrom MG (2017) Engagement and quality of life in under-represented older adults: a community-based participatory research project. Narrative Inquiry in Bioethics 7, E7-E9.

Buffel T (2018) Social research and co-production with older people: developing age-friendly communities. Journal of Aging Studies 44, 52-60.

Buffel T (2019) Older coresearchers exploring age-friendly communities: an 'insider' perspective on the benefits and challenges of peer-research. The Gerontologist 59, 538-548.

Buffel T, Skyrme J and Phillipson C (2017) Connecting research with social responsibility: developing 'age-friendly' communities in Manchester, UK. In Shek D and Hollister R (eds), University Social Responsibility and Quality of Life (Quality of Life in Asia, Vol. 8). Singapore: Springer, pp. 99-120.

Clough R, Green B, Hawes B, Raymond G and Bright L (2006) Older People as Researchers: Evaluating a Participative Project. York, UK: Joseph Rowntree Foundation.

Corrado AM, Benjamin-Thomas TE, McGrath C, Hand C and Laliberte Rudman D (2020) Participatory action research with older adults: a critical interpretive synthesis. The Gerontologist 60, e413-e427.

Dewar BJ (2005) Beyond tokenistic involvement of older people in research - a framework for future development and understanding. International Journal of Older People Nursing in Association with Journal of Clinical Nursing 14, 48-53.

Doran P and Buffel T (2018) Translating research into action: involving older people in co-producing knowledge about age-friendly neighbourhood interventions. Working with Older People 22, 39-47. 
Doyle M and Timonen V (2010) Lessons from a community-based participatory research project: older people's and researchers' reflections. Research on Aging 32, 244-263.

Durose C, Beebeejaun Y, Rees J, Richardson J and Richardson L (2011) Towards Co-production in Research with Communities. Swindon, UK: Connected Communities, Arts and Humanities Research Council.

Ellins J and Glasby J (2016) 'You don't know what you are saying "yes" and what you are saying "no" to': hospital experiences of older people from minority ethnic communities. Ageing \& Society 36, 42-63.

Fenge L-A (2010) Striving towards inclusive research: an example of participatory action research with older lesbians and gay men. British Journal of Social Work 40, 878-894.

Fenge L-A, Fannin A, Armstrong A, Hicks C and Taylor V (2009) Lifting the lid on sexuality and ageing: the experiences of volunteer researchers. Qualitative Social Work 8, 509-524.

Fenge L-A, Jones K and Read R (2010) Connecting participatory methods in a study of older lesbian and gay citizens in rural areas. International Journal of Qualitative Methods 9, 320-333.

Fudge N, Wolfe CDA and McKevitt C (2007) Involving older people in health research. Age and Ageing 36, 492-500.

Greenhalgh T, Jackson C, Shaw S and Janamian T (2016) Achieving research impact through co-creation in community-based health services: literature review and case study. The Milbank Quarterly 94, 392-429.

Gutman C, Hantman S, Ben-Oz M, Criden W, Anghel R and Ramon S (2014) Involving older adults as co-researchers in social work education. Educational Gerontology 40, 186-197.

Hand C, Rudman DL, McGrath C, Donnelly C and Sands M (2019) Initiating participatory action research with older adults: lessons learned through reflexivity. Canadian Journal on Aging/La Revue canadienne du vieillissement 38, 512-520.

Heyn PC, Meeks S and Pruchno R (2019) Methodological guidance for a quality review article. The Gerontologist 59, 197-201.

Hyde P, Burns D, Hassard J and Killett A (2014) Colonizing the aged body and the organization of later life. Organization Studies 35, 1699-1717.

Jones K, Fenge LA, Read R and Cash M (2013) Collecting older lesbians' and gay men's stories of rural life in South-West England and Wales: 'We were obviously gay girls ... (so) he removed his cow from our field'. Forum: Qualitative Social Research 14, 1-21.

Littlechild R, Tanner D and Hall K (2015) Co-research with older people: perspectives on impact. Qualitative Social Work 14, 18-35.

Marsh C (1981) Informants, respondents and citizens. In Bulmer M (ed.), Essays on the History of British Sociological Research. Cambridge: Cambridge University Press, pp. 206-227.

Mays N and Pope C (2000) Assessing quality in qualitative research. British Medical Journal 320, 50-52.

McKillop J and Wilkinson H (2004) Make it easy on yourself!: advice to researchers from someone with dementia on being interviewed. Dementia 3, 117-125.

Mey E and van Hoven B (2019) Managing expectations in participatory research involving older people: what's in it for whom? International Journal of Social Research Methodology 22, 323-334.

Moher D, Liberati A, Tetzlaff J, Altman DG and The PRISMA Group (2009) Preferred reporting items for systematic reviews and meta-analyses: the PRISMA statement. PLOS Medicine 6, e1000097.

Osborn A (1985) Short term funded projects: a creative response to an ageing population? In Butler A (ed.), Ageing. Recent Advances and Creative Responses. London: Croom Helm, pp. 125-136.

Osborn A and Willcocks D (1990) Making research useful and usable. In Peace S (ed.), Researching Social Gerontology: Concepts, Methods and Issues. London: Sage, pp. 188-202.

Pan H, de Donder L, Dury S, Wang R, de Witte N and Verté D (2019) Social participation among older adults in Belgium's Flanders region: exploring the roles of both new and old media usage. Information, Communication \& Society 22, 1956-1972.

Porter G (2016) Reflections on co-investigation through peer research with young people and older people in sub-Saharan Africa. Qualitative Research 16, 293-304.

Pratesi A, Sixsmith J and Woolrych R (2013) Genuine partnership and equitable research: working 'with' older people for the development of a smart activity monitoring system. Innovation Journal 18, 1-17.

Pruchno R (2015) Successful aging: contentious past, productive future. The Gerontologist 55, 1-4.

Ray M (2007) Redressing the balance? The participation of older people in research. In Bernard M and Scharf T (eds), Critical Perspectives on Ageing Societies. Bristol, UK: Policy Press, pp. 73-88. 
Rémillard-Boilard S, Buffel T and Phillipson C (2017) Involving older residents in age-friendly developments: from information to coproduction mechanisms. Journal of Housing for the Elderly 31, 146-159.

Ross F, Donovan S, Brearley S, Victor C, Cottee M, Crowther P and Clark E (2005) Involving older people in research: methodological issues. Health \& Social Care in the Community 13, 268-275.

Shore L, Kiernan L, DeEyto A, Bhaird DNA, Connolly A and White PJ (2018) Older adult insights for age friendly environments, products and service systems. Design and Technology Education: An International Journal 23, 40-58.

Tanner D (2012) Co-research with older people with dementia: experience and reflections. Journal of Mental Health 21, 296-306.

Walker A (2007) Why involve older people in research? Age and Ageing 36, 481-483.

Ward L (2014) Negotiating well-being: older people's narratives of relationships and relationality. Ethics and Social Welfare 8, 293-305.

Ward L and Barnes $\mathbf{M}$ (2016) Transforming practice with older people through an ethic of care. British Journal of Social Work 46, 906-922.

Ward L and Gahagan B (2010) Crossing the divide between theory and practice: research and an ethic of care. Ethics and Social Welfare 4, 210-216.

Ward L, Barnes M and Gahagan B (2011) Alcohol use in later life - older people's perspectives. Quality in Ageing and Older Adults 12, 239-247.

Winter SJ, Rosas LG, Romero PP, Sheats JL, Buman MP, Baker C and King AC (2016) Using citizen scientists to gather, analyze, and disseminate information about neighborhood features that affect active living. Journal of Immigrant and Minority Health 18, 1126-1138.

World Health Organization (2015) World Report on Ageing and Health. Geneva: World Health Organization.

Yankeelov PA, Faul AC, D'Ambrosio JG, Collins WL and Gordon B (2015) 'Another day in paradise': a photovoice journey of rural older adults living with diabetes. Journal of Applied Gerontology 34, 199-218.

Ziegler F and Scharf T (2013) Community-based participatory action research: opportunities and challenges for critical gerontology. In Baars J, Dohmen J, Grenier A and Phillipson C (eds), Ageing, Meaning and Social Structure. Connecting Critical and Humanistic Gerontology. Bristol, UK: Policy Press, pp. 157-180.

Cite this article: James H, Buffel T (2022). Co-research with older people: a systematic literature review. Ageing \& Society 1-27. https://doi.org/10.1017/S0144686X21002014 